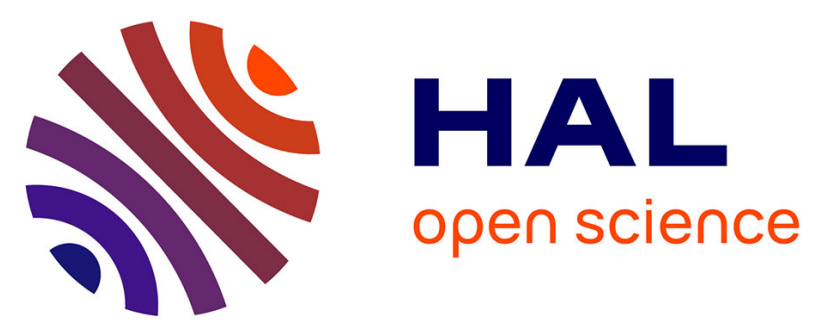

\title{
Développement et validation d'une échelle courte mesurant le désengagement moral en sport (ECDMS)
}

Karine Corrion, Stéphanie Scoffier-Mériaux, Christophe Gernigon, Francois Cury, Fabienne d'Arripe-Longueville

\section{- To cite this version:}

Karine Corrion, Stéphanie Scoffier-Mériaux, Christophe Gernigon, Francois Cury, Fabienne d'ArripeLongueville. Développement et validation d'une échelle courte mesurant le désengagement moral en sport (ECDMS). L'Encéphale, 2010, 10.1016/j.encep.2010.03.003 . hal-02524721

\section{HAL Id: hal-02524721 \\ https://hal.univ-cotedazur.fr/hal-02524721}

Submitted on 3 Apr 2020

HAL is a multi-disciplinary open access archive for the deposit and dissemination of scientific research documents, whether they are published or not. The documents may come from teaching and research institutions in France or abroad, or from public or private research centers.
L'archive ouverte pluridisciplinaire HAL, est destinée au dépôt et à la diffusion de documents scientifiques de niveau recherche, publiés ou non, émanant des établissements d'enseignement et de recherche français ou étrangers, des laboratoires publics ou privés. 
1 Développement et validation d'une Echelle Courte mesurant le Désengagement Moral en Sport (ECDMS)

3

4 Development and Factorial Validity of a Moral Disengagement in Sport Short Scale 5

6

7

8

9

Karine Corrion et Stéphanie Scoffier

Université de Nice Sophia-Antipolis, France

François Cury

CNRS Université de Provence et

Université de Toulon-Sud, France
Christophe Gernigon

Université de Montpellier I,

France

Fabienne d'Arripe-Longueville

Université de Nice Sophia-Antipolis,

France

Adresse de correspondance :

Karine Corrion

Faculté des Sciences du Sport Laboratoire Motricité, Humaine, Education, Santé

Université de Nice Sophia-Antipolis

261, route de Grenoble - BP 3259 06205 Nice cedex 3, France

Téléphone : 0492296532

Fax: 0492296537

Email: corrion.karine@aliceadsl.fr corrion@unice.fr 
1 Résumé

2 L'objet de cette étude était de développer et valider en langue française une Echelle

3 Courte mesurant le Désengagement Moral en Sport en langue française (ECDMS), et de tester

4 son invariance selon le genre. L'outil a été développé sur la base de la littérature existante (22,

5 23). Trois études complémentaires ont été réalisées auprès de 1305 étudiants. Dans la

6 première étude, l'analyse factorielle d'une première version de l'ECDMS a permis d'identifier

7 deux sous-échelles de trois items (i.e., rejet ou partage de responsabilité ; minimisation des

8 transgressions et de leurs conséquences) présentant de bonnes consistances internes. La

9 deuxième étude a confirmé la structure bi-factorielle de l'instrument et a montré son

10 invariance partielle selon le genre. Enfin, la troisième étude a montré la fidélité temporelle et

11 la validité théorique de l'instrument au travers de ses relations avec d'autres construits

12 mesurant l'auto-régulation des affects ou les comportements pro-sociaux des individus (15,

13 16). Au final, l'ECDMS apparaît comme un instrument présentant de bonnes qualités

14 psychométriques et constitue un outil en langue française pouvant ouvrir la voie à de futures

15 recherches sur le désengagement moral d'adolescents ou d'adultes sportifs.

16

17 Mots clés : Fonctionnement moral, transgression, sport, adolescence, validation. 


\section{Development and factorial validity of a Moral Disengagement in Sport Short Scale}

2

Summary. According to Bandura (17), individuals are able to violate their personal standards, without self-sanction, by using the psychological operations of moral disengagement. For Bandura et al. (18), moral disengagement is characterized by eight mechanisms belonging to one of the following four groups: (a) reconstruing conduct, (b) reconsideration of negative effects, (c) disqualification of the victim, and (d) obscuring of personal causal agency. Other researchers have measured moral disengagement in various contexts of everyday life $(19,20,21)$ using the scale from Bandura et al. (18) and suggested that moral disengagement mechanisms would fall into two or three groups according to context. One context in which moral issues have a major role is sport (22). Three complementary studies were carried out with a total of 1305 young French adult athletes to develop and validate a Short French Questionnaire of Moral Disengagement in Sport (SFQMDS) and to test its invariance according to gender. Study $\mathbf{1}-$ With reference to the existing literature $(22,23)$, an initial French version of the SFQDMS was developed. French university students $(\mathrm{N}=220)$ then voluntarily completed the questionnaire. The validity of this preliminary version and the clarity of the items were examined and ascertained, and factorial analyses identified ten items that loaded onto two factors (i.e., Projecting fault onto others or sharing of responsibility; Minimization of transgression and their consequences). Each factor displayed good internal consistency. Study 2 - Confirmatory factor analysis (CFA) was conducted using AMOS 7.0 software. The sample included 1021 French university students $\left(\mathrm{M}_{\mathrm{age}}=21.52 ; \mathrm{SD}=2.34\right)$. The first analysis of the data from 298 French students suggested that four items should be eliminated (15). The six-item model was then tested with a CFA of the data from 723 other participants $\left(\mathrm{M}_{\mathrm{age}}=21.51 ; \mathrm{SD}=2.34\right)$ and exhibited acceptable fit indices: $\left[\chi^{2}(8,723)=1.54 ; \mathrm{p}>0.09 ; \mathrm{GFI}=0.97 ; \mathrm{TLI}=0.97 ; \mathrm{CFI}=\right.$ 0.97 ; RMSEA $=0.03$; RMSEA LO/HI $=0.01 / 0.05]$. These results confirmed the bi-factorial 
1 structure of the instrument, as well as its partial invariance across genders (27) at the most

2 complex level (i.e., strict) of its factorial structure. These statistical analyses demonstrated the

3 excellent internal consistency and very good construct validity of the SFQDMS. Study 3 -

4 The third study examined the temporal stability of the SFQDMS and its theoretical validity

5 with a sample of 221 French students $\left(\mathrm{M}_{\mathrm{age}}=21.00 ; \mathrm{SD}=2.05\right)$. Our results were found to be

6 stable over time. From a theoretical standpoint, the SFQDMS was related to existing

7 instruments that measure individuals' affective self-regulatory efficacy and prosocial behavior

$8(15,16)$. These results demonstrated the external validity of the instrument. Conclusion - The

9 overall results presented in these studies confirmed the good psychometric properties of the

SFQDMS. This questionnaire consists of two subscales of three items measuring two groups

11 of moral disengagement. The first involves projecting the fault for one's own transgressions

12 onto others or sharing of responsibility [e.g., "It's not my fault if I behave badly (cheating or aggression) because it's my opponent who started it"]. The second subscale involves the minimization of transgressions and their consequences (e.g., "It's not serious if I behave badly

15 (cheating or aggression) because I do it to keep the advantage"). This instrument is a reliable

16 tool that could be fruitfully used in future research addressing the moral disengagement of

17 French adolescents or adults in sport. A deeper understanding of the processes involved in moral disengagement would facilitate the development of strategies to prevent or remediate transgressive behaviors in the sport domain.

Key words: Moral functioning, transgressive behavior, sport, adolescence, validation 


\section{Sport (ECDMS)}

\section{INTRODUCTION}

Bien que l'on prône souvent que le sport soit un vecteur de développement de valeurs et d'attitudes morales telles que la loyauté, la responsabilité, le travail en équipe ou le respect des règles (1), la pratique sportive est le terrain de nombreuses transgressions. En premier lieu, l'exacerbation de la logique compétitive génère des comportements de tricherie (2), réduit les comportements pro-sociaux (3) et augmente les comportements anti-sociaux (4). La pratique sportive intensive, notamment à haut niveau, est parfois à l'origine de pathologies physiques et/ou psychiques telles que les troubles des comportements alimentaires (5), et favorise les conduites de transgression, de la simple tricherie jusqu'à la consommation de substances illicites $(6,7)$.

En deuxième lieu, à un niveau compétitif moindre, les individus à la recherche de sensations sont également enclins à franchir les limites des règles établies, jusqu'à prendre des risques inconsidérés, mettant en danger leur vie ou celle d'autrui $(8,9)$. Par exemple, les BASE-jumpers se caractérisent par des scores élevés de recherche de danger et d'aventures, de conduites de consommation de substances illicites et d'accidents. Enfin, les individus présentant des troubles de la personnalité telle la personnalité antisociale (10) ou borderline (11) seraient particulièrement disposés à adopter des comportements transgressifs, dans la vie quotidienne comme dans le contexte sportif $(12,13)$.

Les individus engagés dans ces situations de transgression en contexte sportif présentent tous, au-delà de leurs particularités, un point commun : ils sont incapables de contrôler leurs comportements grâce à des auto-sanctions morales. Ce processus psychologique est désigné, dans la littérature en psychologie sociale, par le concept de « désengagement moral ». Celui-ci désigne le processus qu'un individu utilise pour pouvoir 
1 justifier le bien-fondé de certains actes transgressifs (14). Le désengagement moral se

2 rapporte à un mécanisme autorégulateur qui consiste en une restructuration cognitive de la

3 conduite inhumaine (14). Il est relié négativement à différentes variables psychosociales,

4 telles que les capacités de résistance à la pression sociale négative, les capacités d'auto-

5 régulation des affects, et les comportements prosociaux $(15,16)$.

Les travaux de Bandura $(17,18)$ ont mis en évidence que les individus sont capables

7 de violer leurs standards personnels, sans s'attribuer de sanctions par le biais de huit mécanismes psychologiques caractérisant le désengagement moral, et appartenant aux quatre

9 groupes suivants. Le premier groupe désigne la requalification de la conduite et intègre les mécanismes tels que la justification morale, la comparaison avantageuse, et l'utilisation d'euphémismes. Ces mécanismes consistent en une reconstruction cognitive de la conduite lui permettant de ne pas être considérée comme immorale. Le deuxième groupe désigne la reconsidération des effets négatifs, intégrant la minimisation ou la distorsion des conséquences. Ce mécanisme explique la manière dont les individus peuvent réduire au minimum leur perception des dommages causés par leurs actes. Le troisième groupe désigne la disqualification de la victime et intègre les mécanismes tels que l'attribution de blâme et la déshumanisation. Ils consistent à expliquer comment les victimes sont dévaluées en tant qu'êtres humains ou blâmées. Enfin, le dernier groupe désigne l'obscurcissement du lien causal intégrant les mécanismes tels que le déplacement et la diffusion de responsabilité. Ils consistent à expliquer comment les transgresseurs obscurcissent leur rôle actif dans le mal

21 qu'ils causent par une responsabilité déplacée vers autrui ou partagée. En relation avec ce cadre théorique, ces auteurs (18) ont développé un questionnaire de mesure du désengagement moral comportant 32 items et huit sous-échelles correspondant aux huit mécanismes précités. L'utilisation et l'adaptation de ce questionnaire à différents contextes $(19,20,21)$ suggèrent 
1 que les mécanismes de désengagement moral pourraient se regrouper selon le contexte en

2 deux ou trois groupes de mécanismes. réalisée par Boardley et Kavussanu (22). Ce questionnaire anglo-saxon (i.e., the Moral Disengagement Sport Scale; MDSS) comporte 32 items et six sous-échelles. Les auteurs ont

6 retenu un modèle en six facteurs présentant les indices d'ajustement les plus satisfaisants.

7 Toutefois, ils ont relevé des corrélations élevées entre plusieurs sous-échelles (i.e., entre la requalification de la conduite, la déshumanisation, et l'attribution de blâme, ainsi qu'entre la comparaison avantageuse et la distorsion des conséquences), et suggèrent que la mesure du désengagement moral en sport pourrait impliquer un nombre de facteurs moindre. Une étude qualitative parallèle (23) sur le désengagement moral chez des sportifs français expérimentés a apporté des éléments allant dans le sens de cette hypothèse. Les résultats ont montré que les principaux mécanismes de désengagement moral chez les adolescents concernaient deux grandes catégories. La première concerne le rejet ou le partage de responsabilité avec autrui ». Elle regroupe les mécanismes d'attribution de blâme, de déplacement de responsabilité et de diffusion de responsabilité. La seconde catégorie concerne la minimisation et la requalification de la conduite et de ses conséquences. Elle regroupe la minimisation des conséquences et l'utilisation d'euphémismes. Ces résultats suggèrent donc que le désengagement moral en sport pourrait être mesuré grâce à deux facteurs principaux. Par ailleurs, il n'existe pas à l'heure actuelle d'instrument en langue française mesurant le désengagement moral en sport. L'objet de ce travail était donc sur la base de la littérature existante, de développer et valider en langue française une échelle courte mesurant le désengagement moral en sport (ECDMS) sur une population des deux sexes.

\section{ÉTUDE 1}


L'objectif de cette étude était de proposer une version préliminaire en langue française

2 de l'ECDMS, puis d'en examiner la structure factorielle.

Méthode

Participants et procédure. La population était constituée dans un premier temps de 20 étudiants pour l'analyse de clarté de la version préliminaire, puis de 200 étudiants sportifs volontaires (129 garçons et 71 filles, $\mathbf{M}_{\hat{a g g e}}=21,20$ ans ; $\left.\mathrm{ET}=2,47\right)$ pour l'analyse exploratoire. La passation des questionnaires a respecté les critères de non-obligation, d'anonymat, et de confidentialité des réponses, et s'est déroulée à la fin d'un cours dispensé à la faculté des sciences du sport.

Formulations des items. En se basant sur la littérature existante, un comité d'experts

(chercheurs dans le domaine de la psychologie sociale appliquée au sport) fut chargé de formuler une série d'items permettant d'évaluer le désengagement moral dans le domaine du sport, et plus précisément en sports collectifs. Ces items ont été construits sur la base des verbalisations des athlètes issues de l'étude qualitative du désengagement moral en sport (11), et en relation avec certains items du questionnaire de désengagement moral en sport des versions anglaises longue et courte (MDSS, 22 ; MDSS'S, 24). Une version préliminaire de 22 items fut soumise, lors d'une deuxième étape, à l'analyse de clarté des items sur la population cible. Cette étape a consisté à faire remplir la version exploratoire de l'instrument à chaque sportif (deux groupes de dix sportifs ont été retenus pour les deux sessions organisées) qui devait répondre à chaque item sur une échelle de type Likert en sept points allant de (1) «Pas du tout clair», à (7) « Tout à fait clair». Puis, lors d'un entretien qualitatif individuel, le sportif était invité à justifier les points accordés à chaque item.

\section{Résultats et discussion}

Analyse de clarté. L'analyse des données recueillies lors de la première passation auprès de 10 étudiants $(\mathrm{M}=21 ; \mathrm{ET}=2,15)$ et les commentaires recueillis lors des entretiens 
1 ont engendré des modifications sur deux items (RPR1, MIN3). Une nouvelle analyse de clarté

2 auprès de 10 autres étudiants a permis d'obtenir des scores satisfaisants sur chacune des deux

3 sous-échelles $(\mathrm{M}=4,32 ; \mathrm{ET}=1,12$ et $\mathrm{M}=4,54 ; \mathrm{ET}=1,02)$.

Analyse factorielle exploratoire. La structure factorielle a été examinée par une

analyse factorielle en axes principaux avec rotation Oblimin, en raison des corrélations

6 interfacteurs présumées. Afin d'extraire les facteurs les plus appropriés, une analyse parallèle

7 (25) a été utilisée. Cette méthode d'extraction des facteurs a permis, sans contraindre le

modèle, de mettre en évidence deux facteurs. Ensuite, la structure factorielle en deux facteurs

a été examinée à l'aide d'une analyse factorielle en appliquant une contrainte sur le nombre de

facteurs (i.e., 2). L'observation des coefficients de saturation a permis la sélection, selon la méthode préconisée par Guttman ${ }^{2}$ (26), des 10 items sur 22 présentés dans le tableau I.

L'ensemble des données analysées se sont donc organisées selon un modèle à deux facteurs, expliquant une proportion de variance satisfaisante de $86,43 \%$ (Rejet ou partage de responsabilité, RPR : 47,33\% ; Minimisation, MIN : 39,10\%). $=1,57)$ et $\mathrm{M}_{\mathrm{MIN}}=2,63\left(\mathrm{ET}_{\mathrm{MIN}}=1,52\right)$, et les coefficients Alpha de Cronbach pour les deux sous-échelles étaient satisfaisants $\left(\alpha_{\mathrm{RPR}}=0,84\right.$ et $\left.\alpha_{\mathrm{MIN}}=0,85\right)$. La corrélation inter-échelles observée $(r=0,50)$ montre une relation positive significative mais modérée entre les deux sous-échelles. Des ANOVAs à une voie (sexe) ont mis en évidence des différences significatives entre les scores des garçons et des filles pour l'échelle RPR, F(1, 200) = 9,60, p

$21<0,01\left(\mathrm{M}_{\text {garçons }}=3,06 ; \mathrm{M}_{\text {filles }}=2,65\right)$, ainsi que pour l'échelle $\mathrm{MIN}, \mathrm{F}(1,200)=22,26, \mathrm{p}<$

\footnotetext{
1 Méthode parallèle (25) : extraire les facteurs appropriés sans contraindre le modèle. Au sein de la distribution aléatoire, des valeurs inférieures relatives aux poids des facteurs ont été mises en évidence pour les deux premiers facteurs uniquement [i.e., Facteur 1 (valeur aléatoire $)=1,64,($ valeur de l'ACP $)=8,60 ;$ Facteur $2($ valeur aléatoire $)=1,53 ;($ valeur de l'ACP $)=1,81]$.

${ }^{2}$ Méthode préconisée par Guttman (26) : Les items présentant des coefficients de saturation supérieurs à 0,40 simultanément sur les deux facteurs, ceux dont le coefficient de saturation n'atteignait pas cette valeur sur aucun des deux facteurs, et ceux qui ne saturaient pas sur un facteur regroupant en majorité des items aux contenus sémantiques proches du leur n'ont pas été pris en compte.
} 
$10,001\left(M_{\text {garçons }}=3,18 ; M_{\text {filles }}=2,48\right)$. Ces différences sont consistantes avec la littérature

2 (15), et nous invitent à examiner l'invariance selon le genre de l'instrument.

3 ÉTUDE 2

Les objectifs de cette étude étaient de confirmer la validité factorielle de chaque souséchelle et de tester l'invariance selon le genre.

Méthode

Participants et procédure. La population totale était constituée de 1021 étudiants volontaires pour l'étude (651 garçons et 370 filles, $\mathrm{M}_{\mathrm{âge}}=21,52 ; \mathrm{ET}=2,34$ ), suivant de manière régulière, les cours à la faculté des sciences du sport. Le profil de l'échantillon était similaire à celui de l'étude 1 , ainsi que la procédure de passation du questionnaire. Analyses. Nous avons procédé à plusieurs analyses réalisées à l'aide du logiciel AMOS 7.0 (2) : (a) des analyses factorielles confirmatoires (AFC), (b) un test de l'invariance factorielle du QDMS selon le genre, avec la méthode de Gregorich $^{3}$ (27).

\section{Résultats et discussion} portant sur 298 étudiants (193 garçons et 105 filles, $M_{\hat{a ̂ g e}}=21,00 ; E T=2,03$ ). Selon la méthode préconisée par Guttman (15), les résultats ont suggéré d'éliminer quatre items (i.e., RPR7, RPR8, et MIN1, MIN9), sur les dix $\left[\left(\chi^{2}(34,298)=131,60 ; \mathrm{p}<0,001 ; \mathrm{GFI}=0,92\right.\right.$; $\mathrm{TLI}=0,92 ; \mathrm{CFI}=0,94 ; \mathrm{RMSEA}=0,09)]$. Le modèle à six items répartis en deux facteurs ainsi obtenu, a ensuite été soumis à une nouvelle AFC auprès de 723 autres étudiants sportifs $\left(\mathrm{M}_{\mathrm{a} g e}=21,51 ; \mathrm{ET}=2,34\right)$. Ce modèle à six items s'est avéré significativement ajusté aux données, avec des indices d'ajustement plus satisfaisant, que celui obtenu dans la première analyse à 10 items $\left[\left(\chi^{2}(8,723)=1,54 ; \mathrm{p}>0,09 ; \mathrm{GFI}=0,97 ; \mathrm{TLI}=0,97 ; \mathrm{CFI}=0,97\right.\right.$;

RMSEA $=0,03 ;$ RMSEA LO/HI = 0,01/0,05)]. Les coefficients Alpha de Cronbach, étaient

\footnotetext{
${ }^{3}$ Méthode de Gregorich (27) : Cette méthode nécessite que la structure factorielle du modèle soit de plus en plus contrainte (i.e., modèle sans contrainte, Dimensional ; modèle contraint au niveau des scores de régression, Metric ; au niveau des constantes, Strong/Scalar; au niveau des erreurs de mesure, Strict)
} 
1 satisfaisants pour les deux sous-échelles $\left(\alpha_{R P R}=0,76\right.$ et $\left.\alpha_{M I N}=0,83\right)$. Une corrélation positive

2 significative a été observée entre les deux sous échelles $(\mathrm{r}=0,62, \mathrm{p}<0,001)$. Invariance selon le genre. Des analyses d'invariance selon le genre ont été effectuées

4 sur le même échantillon de 723 étudiants, à partir de la méthode de ré-échantillonnage

5 Bootstrap ${ }^{4}$. Des $\mathrm{AFC}^{5}$ ont été réalisées pour chaque modèle. Cette première série d'analyses

6 de l'échantillon soutient l'invariance partielle au niveau le plus contraint (i.e., strict) de la

7 structure factorielle du ECDMS à travers le genre, et montre que cet instrument est valide

8 pour les garçons et les filles.

9 ÉTUDE 3

L’objectif de cette étude était d'évaluer la fidélité temporelle de l'instrument et sa validité théorique au travers de ses liens avec d'autres instruments. La littérature montre en effet que le désengagement moral est relié négativement aux capacités d'auto-régulation des affects négatifs (contrôle de ses humeurs, contrôle des réponses aux agressions) et aux comportements pro-sociaux $(15,16)$.

Méthode

Participants et procédure. La population était constituée de 221 étudiants sportifs volontaires $\left(\mathrm{M}_{\mathrm{a} g e}=21,00 ; \mathrm{ET}=2,05\right)$. Le profil de l'échantillon était globalement similaire à celui des études précédentes, ainsi que la procédure de passation du questionnaire. La fidélité temporelle de l'outil a été éprouvée au travers d'une mesure répétée deux fois sur le même échantillon avec un intervalle de quatre semaines.

\footnotetext{
${ }^{4}$ Méthode de ré-échantillonnage Bootstrap : Cette méthode est basée sur un ré-échantillonnage des données à partir d'une distribution estimée de la population réelle ou échantillon observé.

${ }^{5}$ Les tests de $\chi^{2}$ étaient significatifs, mais tous les ratios $\chi^{2} / \mathrm{ddl}$ étaient inférieurs à 2 . Comme préconisé par Byrne (28), les indices CFI, TLI et RMSEA étaient satisfaisants (i.e., > 0,90 pour CFI et TLI ; < 0,06 pour RMSEA), pour les différents modèles, ainsi que les différences avec le modèle sans contrainte (i.e., dimensional). En examinant les différences entre les modèles contraints (i.e.. Metric, Strong/Scalar et Strict) et le modèle sans contrainte (i.e., dimensional), les modèles Metric et Strong/Scalar n'étaient pas sensiblement différents. Cependant, le model 4 (Strict) montre un ajustement plus pauvre (Chi2/ddl $=64,85, \mathrm{p}<0,01)$, et le delta Chi2 était significatif entre le modèle Strong/Scalar et Strict. Ainsi, le logiciel AMOS 7.0 a suggéré des indices de modifications pour l'équivalence des groupes selon le genre, contraints au niveau des erreurs de mesure pour certains items. Le cinquième modèle non contraint au niveau des erreurs de mesures (i.e., items MIN8, MIN10 et RPR5), dans les deux groupes de genre, a montré une invariance partielle (i.e., adéquation des données des échantillons de garçons et de filles similaires à l'exception d'une ou plusieurs erreurs de mesure) au niveau strict.
} 
Mesures. Les capacités d'auto-régulation des affects négatifs ont été évaluées en

2 utilisant l'échelle validée en langue française (29) du « Social Skills Rating System » (SSRS,

3 30). Les comportements prosociaux ont été évalués à l'aide de la version canadienne française

$4 \mathrm{du}$ «Toddler Behavior Assessment Questionnaire » (31), et le désengagement moral a été

5 mesuré grâce au questionnaire précédemment développé. Les consistances internes de

$6 \quad$ l'ensemble des échelles étaient satisfaisantes, et se situaient entre 0,84 et 0,92.

\section{$7 \quad$ Résultats}

La fidélité temporelle de la structure du questionnaire a d'abord été vérifiée à l'aide de tests $t$ de Student pour échantillons appariés afin de vérifier la non-différence de score à la fois dans le questionnaire au temps T1 et T2, mais également par rapport à zéro. Les tests $t$ se sont avérés non significatifs pour les deux sous-échelles, ce qui témoigne de l'absence de différence significative entre les structures obtenues à chacune des deux occasions de mesure. En complément, l'analyse des corrélations, nous a permis de confirmer la stabilité temporelle des sous-échelles au temps T1 et T2. Les scores obtenus ( $r$ de Bravais Pearson) étaient pour RPR de 0,66 et pour MIN de 0,74 ( $<<0,01$ ). Enfin, la consistance des deux sous-échelles étaient satisfaisantes au temps T1 et T2 (RPR : $\alpha_{T l}=0,81$ et $\alpha_{T 2}=0,83 ;$ MIN : $\alpha_{T l}=0,88$ et $\alpha_{T 2}$ $17=0,91)$.

Des analyses de régressions entre les différentes variables (au temps T1) ont permis de mettre en évidence différentes relations significatives consistantes avec la littérature (6 15, 16). Les sous-échelles de désengagement moral sont en effet apparues négativement reliées à la sous-échelle des capacités d'auto-régulation des affects négatifs ( $\beta=-0,45, p<$ $0,01)$, et à la sous-échelle des comportements prosociaux $(\beta=-024, p<0,01)$. Ainsi, conformément à la littérature $(15,16)$, moins les individus ont de capacités d'auto-régulation des affects et de comportements prosociaux, et plus ils se désengagent moralement. 
Cette étude montre des éléments de fidélité et de validité satisfaisants de l'ECDMS.

2 Cet instrument est composé de deux sous-échelles de trois items chacun, mesurant deux

3 groupes de mécanismes de désengagement moral en sport. Ces résultats témoignent de la

4 validité du regroupement dans le contexte sportif de certains mécanismes définis par Bandura

5 dans la vie quotidienne (18). Les items relatifs au facteur « Rejet ou partage de

6 responsabilité » regroupent l'idée que l'on rejette la faute sur autrui pour justifier ses

7 transgressions, ou que l'on partage cette faute avec autrui. En premier lieu, ce rejet de la faute

8 ou de la responsabilité sur l'autre est justifié, par la pression subie par l'athlète de la part

9 d'autres acteurs (i.e., entraîneur, arbitre, adversaire, spectateur ou partenaire), correspondant

10 au mécanisme de déplacement de responsabilité. En second lieu, il se justifie par la

11 responsabilité partagée de la transgression avec autrui (i.e., diffusion de responsabilité), ainsi

12 que par l'attribution de la faute à ces mêmes acteurs comme une réaction de défense justifiable (i.e., attribution de blâme). Les items relatifs au facteur « Minimisation » correspond au mécanisme de minimisation ou de distorsion des conséquences définis par Bandura et al. (18). Ce facteur de la minimisation des transgressions et leurs conséquences correspond à l'idée que l'individu cherche à diminuer la gravité des transgressions qu'il effectue en se rapportant à la culture spécifique du sport (i.e., habitude de transgresser en compétition), ainsi qu'aux enjeux compétitifs (21). Les items se référant à l'utilisation d'euphémismes faisant initialement partie de ce facteur (23) n'ont pas été retenus suite aux résultats de l'analyse factorielle. En outre, nos résultats montrent l'invariance partielle de la

21 structure factorielle du désengagement moral en sport à travers le genre au niveau le plus 22 contraint. L'ECDMS est donc un instrument valide pour les garçons et les filles. La validité de construit de l'instrument pourra être enrichie en examinant ses relations avec d'autres variables pertinentes d'un point de vue théorique. Cette étude sera facilitée par la nature écourtée de l'échelle, comparativement aux instruments anglophones comportant 32 
1 items $(18,22)$. Ainsi, les relations entre le désengagement moral et l'efficacité auto-

2 régulatrice relative à l'évitement des pressions sociales négatives et le comportement

3 transgressif lui-même (15), pourraient être examinées. Plus généralement, des travaux

4 ultérieurs pourront s'attacher à examiner la contribution des mécanismes de désengagement

5 moral dans la prédiction des comportements de transgression en contexte sportif. Bandura et

6 al. (15) précisaient que le désengagement moral favorise l'adoption de comportements

7 déviants ou transgressifs, comme l'agression physique et verbale, la fraude, ou la

8 consommation de drogue ou d'alcool. Des travaux futurs devront donc tenter de mieux

9 identifier les antécédents tant individuels (e.g., traits et troubles de la personnalité, recherche

10 de sensation) que contextuels (climat familial ; relations avec l'entraîneur) qui font que

11 l'individu est capable de violer ses standards personnels sans s'attribuer de sanctions. La

12 tricherie, et d'autres comportements déviants tels que le dopage se produisent avec une

13 fréquence alarmante en contexte sportif $(33,34)$. De même qu'il est commun de dire qu'un

14 adolescent a sombré dans la délinquance «à cause de ses mauvaises fréquentations », l'athlète

15 est confronté à de puissants phénomènes de pression sociale, qui nécessite des compétences

16 d'auto-régulation affirmées. La meilleure compréhension de ces mécanismes d'auto-

17 régulation devrait rendre possible l'élaboration de stratégies de prévention ou de remédiation

18 des conduites transgressives dans ce contexte spécifique.

En conclusion, l'ECDMS présente des propriétés psychométriques satisfaisantes, et,

20 peut être utilisée auprès d'une population francophone. Cette échelle est un instrument utile

21 pour comprendre comment cette population se justifie moralement face à certains actes

22 transgressifs dans le contexte sportif, et pourra être utilisée en relation avec d'autres construits 23 psychologiques, et faciliter la prévention des transgressions. 


\section{Références}

2 1. Sage G. Power and ideology in American Sport. A critical perspective. Champaign, IL:

$3 \quad$ Human Kinetics ; 1990

4 2. Orlick T. Winning through cooperation: Competitive insanity, cooperative alternatives. Washington, DC: Acropolis Press ; 1978.

3. Kleiber DA, Roberts GC. The effects of sport experience in the development of social character: A preliminary investigation. J Sport Exerc Psychol 1981 ; 3 : 114-122.

4. Kohn A. No contest: The case against competition. Boston: Houghton Mifflin ; 1986.

5. Petrie TA, Greenleaf CA. Eating disorders in sport: From theory to research to intervention. In G. Tenenbaum (Ed.), Handbook of Sport Psychology, 3rd Edition (pp. 352-378). J. Wiley \& Sons, Inc, Hoboken, New Jersey ; 2007.

6. Long T, Pantaléon N, Bruant G, et al. A qualitative study of moral reasoning of young elite athletes. The Sport Psychologist $2006 ; 20: 330-347$.

7. Lucidi F, Zelli A, Mallia L, et al. The social-cognitive mechanisms regulating adolescents' use of doping substances. J Sports Sci 2008 ; 26 : 447-456

8. Michel G, Cazenave N, Delpouve C, et al. Profils de personnalité et fonctionnement émotionnel dans les sports extrêmes : à propos d'une étude exploratoire chez des BASEjumpers. Ann Méd Psychol 2009 ; 167 : 72-77.

9. Patel DR, Luckstead EF. Sport participation, risk taking and health risk behaviors. Adolescent Medecine $2000 ; 11: 141-155$.

10. Gunderson JG, Zanarini MC. Pathogenesis of bordeline personality disorder. In: Tasman A, Hales RE, Frances AJ, editors. American Psychiatry Press Review of Psychiatry, 8. Washington DC: American Psychiatric Press ; 1989, p 25-48. 
1 11. Brooner RK, Herbst JH, Schmidt CW, et al. Antisocial personality among drug users:

2 relations to others personality disorders and to the five-factors model of personality. J

$3 \quad$ Nerv Ment Dis $1993 ; 18: 313-319$.

4 12. Bénézech M, Le Bihan, et Bourgeois ML. Criminologie et psychiatrie. Encycl Méd Chir, $5 \quad$ Psychiatrie $2002 ; 37-906-A-10$.

6 13. Purper-Ouakil D, Michel G, Baup N, et al. Aspects psychopathologiques de l'exercice 7 physique intensif chez l'enfant et l'adolescent : mise au point à partir d'une situation clinique. Ann Méd Psychol $2002 ; 160$ : 543-549.

14. Bandura A. Moral disengagement in the perpetration of inhumanities. Pers Soc Psychol Rev 1999 ; $3: 193-209$

15. Bandura A, Caprara GV, Barbaranelli C, et al. Sociocognitive self-regulatory mechanisms governing transgressive behavior. J Pers Soc Psychol 2001 ; 80 : 125-135.

16. Bandura A, Caprara GV, Barbaranelli C, et al. Role of affective self-regulatory efficacy in diverse spheres of psychosocial functioning. Child Devel $2003 ; 74$ : 769-782.

17. Bandura A. Social cognitive theory of moral thought and action. In WM Kurtines \& JL Gewirtz (Eds.), Handbook of moral behavior and development: Theory, research, and applications (Vol. 1, pp. 71-129). Hillsdale, NJ: Erlbaum ; 1991.

18. Bandura A, Barbaranelli C, Caprara GV, et al. Mechanisms of moral disengagement in the exercise of moral agency. J Pers Soc Psychol 1996 ; 71 : 364-374.

19. Aquino K, Reed II A, Thau, S, et al. A grotesque and dark beauty: How moral identity and mechanisms of moral disengagement influence cognitive and emotional reactions to war. $\mathbf{J}$ Exp Soc Psychol 2007 ; 43 : 385-392.

20. Caprara GV, Capanna C. Moral civic disengagement and values. Ricerche di Psicologia 2005 ; 28 : 67-84. 
1 21. Moore C. Moral disengagement in process of organizational corruption. J business ethics $2007 ; 80: 129-139$.

22. Boardley ID, Kavussanu M. Development and validation of the moral disengagement in sport scale. J Sport Exerc Psychol 2007 ; 29 : 608-628.

23. Corrion K, Long T, Smith AL, et al. «It's not my fault; It's not serious »: Athlete accounts of moral disengagement in competitive sport. The Sport Psychologist, $2009 ; 23$ : 388-404.

24. Boardley ID, Kavussanu M. The Moral Disengagement in Sport Scale - Short. J Sports Sci $2008 ; 26: 1507-1517$.

25. Horn JL. A rationale and test for the number of factors in factors analysis. Psychometrika $1965 ; 30: 179-185$.

26. Guttman L. Some necessary conditions for common factors analysis. Psychometrika 1954 ; $19: 149-185$

27. Gregorich SE. Do self-report instruments allow meaningful comparisons across diverse population groups? Testing measurement invariance using the confirmatory factor analysis framework. Med Care 2006 ; 44 : 78-94.

28. Byrne B. Factor analytic models: Viewing the structure of an assessment instrument from three perspectives. J Pers Assess 2005 ; 85: 17-32.

29. Fortin L, Royer E, Marcotte D, et al. Epreuves de validité d'une mesure d'habiletés sociales auprès d'adolescents québécois à l'école secondaire. Psychologie et Psychométrie $2001 ; 22: 23-43$.

30. Gresham FM, Elliot SN. Social skills rating system. American Guidance Service, Psy Can, Toronto, Canada ; 1990. 
1 31. Lemelin JP. Le questionnaire d'évaluation du comportement de l'enfant : version

2 canadienne française du Toddler Behavior Assessment Questionnaire. Can J Behav Sci $3 \quad 2007 ; 39: 291-300$.

4 32. Arbuckle JL. AMOS 7.0 User's Guide. Amos Development Corporation, Spring House, $5 \quad$ PA $; 2006$.

6 33. Kavussanu M. Motivational predictors of prosocial and antisocial behavior in football. J $7 \quad$ Sports Sci $2006 ; 24: 575-588$.

8 34. Shields D, Bredemeier B, LaVoi N, et al. The behavior of youth, parents, and coaches:

9 The good, the bad, and the ugly. Journal of Research on Character Education $2005 ; 3$ : $10 \quad 43-59$. 
TABLEAU I. - Saturations factorielles de chaque item sur les facteurs considérés 2

\begin{tabular}{|c|c|c|c|c|c|c|c|c|}
\hline & \multicolumn{2}{|c|}{$\begin{array}{l}\text { ETUDE } 1 \\
(n=200)\end{array}$} & \multicolumn{2}{|c|}{$\begin{array}{l}\text { ETUDE } 2 a \\
(n=298)\end{array}$} & \multicolumn{2}{|c|}{$\begin{array}{r}\text { ETUDE } 2 b \\
(n=723)\end{array}$} & \multicolumn{2}{|c|}{$\begin{array}{l}\text { ETUDE 2c } \\
(n=723)\end{array}$} \\
\hline & F 1 & F 2 & $\mathrm{~F} 1$ & F 2 & $\mathrm{~F} 1$ & F2 & Garçons & Filles \\
\hline $\begin{array}{l}\text { RPR1. Ce n'est pas de ma faute, si je me comporte mal (tricherie ou } \\
\text { agression), quand mes coéquipiers exercent une pression sur moi }\end{array}$ & 0,52 & $6,29^{\mathrm{E}}-02$ & 1 & I & I & / & I & I \\
\hline $\begin{array}{l}\text { RPR2. Ce n'est pas de ma faute, si je me comporte mal (tricherie ou } \\
\text { agression), quand un adversaire ne respecte pas les règles du jeu }\end{array}$ & 0,25 & 0,39 & / & I & / & / & / & / \\
\hline $\begin{array}{l}\text { RPR3. Ce n'est pas de ma faute, si je me comporte mal (tricherie ou } \\
\text { agression), quand c'est mon entraîneur qui me le demande }\end{array}$ & 0,63 & $8,57^{\mathrm{E}}-02$ & / & I & / & / & / & / \\
\hline $\begin{array}{l}\text { RPR4. Ce n'est pas de ma faute, si je me comporte mal (tricherie ou } \\
\text { agression), quand c'est pour donner une leçon à l'adversaire }\end{array}$ & 0,33 & 0,20 & / & / & / & / & / & / \\
\hline $\begin{array}{l}\text { RPR5. Ce n'est pas de ma faute, si je me comporte mal (tricherie ou } \\
\text { agression), quand l'arbitre ne siffle presque aucune faute de l'équipe } \\
\text { adverse }\end{array}$ & 0,16 & 0,82 & I & $\mathbf{0 , 8 0}$ & 0,35 & $\mathbf{0 , 8 1}$ & 0,81 & 0,81 \\
\hline $\begin{array}{l}\text { RPR6. Ce n'est pas de ma faute, si je me comporte mal (tricherie ou } \\
\text { agression), quand je n'ai qu'une petite part de responsabilité dans le } \\
\text { tort commis par l'équipe }\end{array}$ & 0,27 & 0,26 & I & I & I & I & I & I \\
\hline $\begin{array}{l}\text { RPR7. Ce n'est pas de ma faute, si je me comporte mal (tricherie ou } \\
\text { agression), quand c'est l'adversaire qui a commencé en premier }\end{array}$ & $7,00^{\mathrm{E}}-02$ & 0,63 & I & 0,77 & I & I & / & I \\
\hline $\begin{array}{l}\text { RPR8. Ce n'est pas de ma faute, si je me comporte mal (tricherie ou } \\
\text { agression), quand l'arbitre est injuste }\end{array}$ & $2,91^{\mathrm{E}}-03$ & 0,76 & I & 0,70 & I & I & I & I \\
\hline $\begin{array}{l}\text { RPR9. Ce n'est pas de ma faute, si je me comporte mal (tricherie ou } \\
\text { agression), quand le coach exerce une pression sur moi }\end{array}$ & 0,44 & 0.10 & / & I & I & / & I & I \\
\hline $\begin{array}{l}\text { RPR10. Ce n'est pas de ma faute, si je me comporte mal (tricherie ou } \\
\text { agression), quand personne ne respecte les règles du jeu }\end{array}$ & $5,55^{\mathrm{E}}-02$ & 0,59 & I & 0,74 & 0,47 & 0,83 & 0,81 & 0,85 \\
\hline $\begin{array}{l}\text { RPR11. Ce n'est pas de ma faute, si je me comporte mal (tricherie ou } \\
\text { agression), quand tout le monde dans l'équipe le fait }\end{array}$ & 0,60 & $6,70^{\mathrm{E}}-03$ & I & I & I & I & I & I \\
\hline $\begin{array}{l}\text { RPR12. Ce n'est pas de ma faute, si je me comporte mal (tricherie ou } \\
\text { agression), quand l'adversaire fait des coups en douce }\end{array}$ & $5,64^{\mathrm{E}}-02$ & 0,74 & I & 0,78 & 0,39 & 0,83 & $\mathbf{0 , 8 3}$ & $\mathbf{0 , 8 3}$ \\
\hline $\begin{array}{l}\text { MIN1. Ce n'est pas grave, si je me comporte mal (tricherie ou } \\
\text { agression), quand c'est pour pousser l'adversaire à la faute, c'est le jeu }\end{array}$ & 0,62 & $\begin{array}{l}7,03^{\mathrm{E}}- \\
02\end{array}$ & 0,81 & I & I & I & I & I \\
\hline $\begin{array}{l}\text { MIN2. Ce n'est pas grave, si je me comporte mal (tricherie ou } \\
\text { agression), quand c'est pour empêcher l'adversaire de reprendre } \\
\text { l'avantage, c'est le jeu }\end{array}$ & 0,74 & $6,42^{\mathrm{E}}-02$ & 0,83 & / & $\mathbf{0 , 8 7}$ & 0,43 & 0,86 & $\mathbf{0 , 8 5}$ \\
\hline $\begin{array}{l}\text { MIN3. Ce n'est pas grave, si je me comporte mal (tricherie ou } \\
\text { agression), quand c'est une tactique de jeu pour gagner du temps }\end{array}$ & 0,43 & 0,32 & I & / & I & I & / & / \\
\hline $\begin{array}{l}\text { MIN4. Ce n'est pas grave, si je me comporte mal (tricherie ou } \\
\text { agression), quand je n'utilise que les limites du règlement pour gagner }\end{array}$ & 0,21 & 0,25 & I & I & I & I & I & I \\
\hline $\begin{array}{l}\text { MIN5. Ce n'est pas grave, si je me comporte mal (tricherie ou } \\
\text { agression), quand c'est pour gêner l'adversaire, cela fait partie du jeu }\end{array}$ & 0,41 & 0,29 & / & / & / & / & / & / \\
\hline $\begin{array}{l}\text { MIN6. Ce n'est pas grave, si je me comporte mal (tricherie ou } \\
\text { agression), quand c'est pour déstabiliser l'adversaire }\end{array}$ & 0,51 & 0,24 & / & / & / & I & / & / \\
\hline $\begin{array}{l}\text { MIN7. Ce n'est pas grave, si je me comporte mal (tricherie ou } \\
\text { agression), quand c'est pour pousser l'adversaire à la faute en fin de } \\
\text { match, et gagner du temps }\end{array}$ & 0,46 & 0,33 & I & I & I & I & / & I \\
\hline $\begin{array}{l}\text { MIN8. Ce n'est pas grave, si je me comporte mal (tricherie ou } \\
\text { agression), quand c'est pour gagner }\end{array}$ & 0,74 & $2,98^{\mathrm{E}}-02$ & 0,75 & I & $\mathbf{0 , 8 7}$ & 0,44 & 0,86 & 0,84 \\
\hline $\begin{array}{l}\text { MIN9. Ce n'est pas grave, si je me comporte mal (tricherie ou } \\
\text { agression), quand c'est pour garder l'avantage }\end{array}$ & 0,82 & $3,40^{\mathrm{E}}-02$ & 0,63 & I & I & / & I & I \\
\hline $\begin{array}{l}\text { MIN10. Ce n'est pas grave, si je me comporte mal (tricherie ou } \\
\text { agression), car tous les moyens sont bons pour parvenir à la victoire }\end{array}$ & 0,75 & $2,92^{\mathrm{E}}-02$ & 0,83 & / & $\mathbf{0 , 8 7}$ & 0,39 & $\mathbf{0 , 8 7}$ & 0,83 \\
\hline
\end{tabular}

4 Notes. RPR ; «Rejet ou partage de responsabilité »= Déplacement et Diffusion de responsabilité, et Attribution

5 de blâme ; MIN ; «Minimisation »= Minimisation des conséquences. 


\section{Echelle Courte de Désengagement Moral en Sport (ECDMS)}

Les affirmations qui suivent sont à replacer au cours de situations que vous avez rencontrées dans la pratique de votre sport. Pour chacune d'elles, dites si vous êtes :

\begin{tabular}{|c|c|c|c|c|c|}
\hline $\begin{array}{c}\text { Pas du } \\
\text { tout } \\
\text { d'accord } \\
1\end{array}$ & $\begin{array}{c}\text { Pas } \\
\text { d'accord }\end{array}$ & $\begin{array}{c}\text { Pas trop } \\
\text { d'accord }\end{array}$ & $\begin{array}{c}\text { Un peu } \\
\text { d'accord }\end{array}$ & $\begin{array}{c}\text { Assez } \\
\text { d'accord }\end{array}$ & $\begin{array}{c}\text { Tout à } \\
\text { fait } \\
\text { d'accord } \\
6\end{array}$ \\
\hline
\end{tabular}

\begin{tabular}{|c|c|c|c|c|c|c|}
\hline $\begin{array}{l}\text { 1) Ce n'est pas grave, si je me } \\
\text { comporte (tricherie ou } \\
\text { agression), quand c'est pour } \\
\text { gagner }\end{array}$ & 1 & 2 & 3 & 4 & 5 & 6 \\
\hline $\begin{array}{l}\text { 2) Ce n'est pas de ma faute, si je } \\
\text { me comporte (tricherie ou } \\
\text { agression), quand personne ne } \\
\text { respecte les règles du jeu }\end{array}$ & 1 & 2 & 3 & 4 & 5 & 6 \\
\hline $\begin{array}{l}\text { 3) Ce n'est pas grave, si je me } \\
\text { comporte (tricherie ou } \\
\text { agression), quand c'est pour } \\
\text { empêcher l'adversaire de } \\
\text { reprendre l'avantage, c'est le } \\
\text { jeu }\end{array}$ & 1 & 2 & 3 & 4 & 5 & 6 \\
\hline $\begin{array}{l}\text { 4) Ce n'est pas de ma faute, si je } \\
\text { me comporte (tricherie ou } \\
\text { agression), quand l'arbitre ne } \\
\text { siffle presque aucune faute de } \\
\text { l'équipe adverse }\end{array}$ & 1 & 2 & 3 & 4 & 5 & 6 \\
\hline $\begin{array}{l}\text { 5) Ce n'est pas grave, si je me } \\
\text { comporte (tricherie ou } \\
\text { agression), car tous les moyens } \\
\text { sont bons pour parvenir à la } \\
\text { victoire }\end{array}$ & 1 & 2 & 3 & 4 & 5 & 6 \\
\hline $\begin{array}{l}\text { 6) Ce n'est pas de ma faute, si je } \\
\text { me comporte (tricherie ou } \\
\text { agression), quand l'adversaire } \\
\text { fait des coups en douce }\end{array}$ & 1 & 2 & 3 & 4 & 5 & 6 \\
\hline
\end{tabular}

Notes : Les items suivants mesurent respectivement, le rejet ou le partage de responsabilité : 2, 4, 6 et la Minimisation:

$1,3,5$.

(C) Karine Corrion, Stéphanie Scoffier, Christophe Gernigon, François Cury, \& Fabienne d'Arripe-Longueville 
